\title{
IN VITRO REACTIVATION OF HUMAN IMMUNODEFICIENCY VIRUS-1 UPON STIMULATION WITH SCRUB TYPHUS RICKETTSIAL INFECTION
}

\author{
MASAKO MORIUCHI, AKIRA TAMURA, AND HIROYUKI MORIUCHI \\ Department of Molecular Microbiology and Immunology, Nagasaki University Graduate School of Biomedical Sciences, Nagasaki, \\ Japan; Department of Microbiology, Niigata College of Pharmacy, Niigata, Japan
}

\begin{abstract}
While a number of microbial infections induce a transient burst in viral load in individuals infected with human immunodeficiency virus-1 (HIV-1), a recent study has suggested that scrub typhus may suppress HIV-1 infection. We investigated the effects of Orientia tsutsugamushi on HIV-1 infection. In vitro HIV-1 infection experiments were conducted using peripheral blood mononuclear cells (PBMC) acutely infected with R5 and X4 HIV-1 or PBMC derived from patients receiving highly active antiretroviral therapy (HAART) whose plasma viral load was undetectable. Stimulation of PBMC with $O$. tsutsugamushi induced production of proinflammatory cytokines and $\beta$-chemokines, and markedly down-regulated expression of CCR5. Although pretreatment with O. tsutsugamushi rendered PBMC resistant to R5 HIV-1, it otherwise enhanced HIV-1 replication. Stimulation by $O$. tsutsugamushi induced HIV-1 replication in PBMC from patients receiving HAART. These findings suggest that scrub typhus does not necessarily suppress HIV-1 infection and does have potential to enhance HIV-1 replication.
\end{abstract}

\section{INTRODUCTION}

Immune activation or perturbation of cytokine networks following microbial coinfections generally increases human immunodeficiency virus-1 (HIV-1) viremia. However, Watt and others have recently reported the possible HIV-1inhibitory effects of scrub typhus, ${ }^{1}$ which is an acute febrile disease that is endemic in tropical Asia where HIV-1 infection is also prevalent. Their hypothesis is intriguing but also remains somehow speculative, partly because of difficulty in conducting a prospective and well-controlled clinical study in Thailand, as well as a lack of precise laboratory data supporting their hypothesis. We investigated effects of Orientia tsutsugamushi infection on in vitro HIV-1 infection of peripheral blood mononuclear cells (PBMC).

\section{MATERIALS AND METHODS}

Cells. The PBMC were isolated from healthy volunteers who were not infected with HIV, as described previously. ${ }^{2}$ Resting $\mathrm{CD}^{+}{ }^{+} \mathrm{T}$ cells were isolated from HIV-1-infected individuals whose plasma viral RNA was undetectable upon receiving highly active antiretroviral therapy (HAART), as described previously. ${ }^{3,4}$

Viruses and rickettsiae. Virus stocks were prepared by transfecting 293 cells with the following HIV-1 infectious molecular clones: ${ }^{2}$ AD8 (subtype B, R5), YU-2 (subtype B, R5), NL4-3 (subtype B, X4), ELI1 (subtype B, X4), 89.6 (subtype $\mathrm{B}, \mathrm{R} 5 \mathrm{X} 4$ ), and 93JP-NH1 (subtype E, R5X4). The proinflammatory cytokines tumor necrosis factor- $\alpha$ (TNF- $\alpha)$ and interleukin-1 $\beta$ (IL-1 $\beta$ ), as well as the $\beta$-chemokines regulated upon activation normal $\mathrm{T}$ cell expressed and secreted (RANTES) protein and macrophage inflammatory protein$1 \beta$, were not detected in these virus stocks.

Cell-free stocks of $O$. tsutsugamushi strains Karp or Kuroki were propagated in L929 cells. ${ }^{5}$ In certain experiments, $O$. tsutsugamushi stocks were pretreated at $56^{\circ} \mathrm{C}$ for 60 minutes. Rickettsial replication was not detected by the $\mathrm{L} 929$ cell culture method.

Infections. For acute infection experiments, PBMC derived from healthy volunteers were mock-infected or infected with cell-free stocks of $O$. tsutsugamushi strains Karp or Kuroki, and then infected with HIV-1 stocks. Inocula were standard- ized by reverse transcriptase (RT) activity $(10,000 \mathrm{cpm}$ per 4 $\times 10^{5}$ cells, as determined by RT assays). Viral replication was monitored by RT activity in cell-free culture supernatants. ${ }^{2}$ For reactivation experiments, PBMC derived from patients receiving HAART were mock-infected or infected with the Karp strain, and viral replication was monitored by measuring p24 antigen levels in cell-free culture supernatants., ${ }^{3,4}$

Single-round viral replication assays. A replicationincompetent luciferase-reporter recombinant virus NL4-3luc$\mathrm{R}^{-} \mathrm{E}^{-}$was pseudotyped with envelope (env) protein from R5 HIV-1 JRFL or X4 HIV-1 HXB2. ${ }^{2}$ The PBMC were not treated, pretreated, or post-treated with the Karp strain (live or heat-inactivated) or supernatants from O. tsutsugamushi (Karp strain)-infected autologous PBMC, and luciferase activity in the infected cell lysates was determined. ${ }^{2}$

Flow cytometry. Cell surface expression of CCR5 and CXCR4 was demonstrated by staining cells with phycoerythrin-conjugated anti-CCR5 monoclonal antibody 2D7 and anti-CXCR4 monoclonal antibody 12G5 (R\&D Systems, Minneapolis, MN) and analyzed by FACScan (BectonDickinson Immunocytochemistry Systems; San Jose, CA). ${ }^{2}$

Transient expression assays. Forty million PBMC were transfected with $40 \mu \mathrm{g}$ of pGL-HIV-1-LTR (a luciferase reporter under the control of the HIV-1 long terminal repeat [LTR]) along with $10 \mu \mathrm{g}$ of pSV2-CAT or pSV2-Tat (encoding HIV-1 Tat), and luciferase activity in the transfected cells was determined, as described previously. ${ }^{2}$

Enzyme-linked immunosorbent assay (ELISA). Levels of cytokines were determined by commercially available ELISA kits, according to the manufacturer's instruction (R\&D Systems).

\section{RESULTS}

Dichotomous effects of $O$. tsutsugamushi on in vitro HIV-1 infection. In acute HIV-1 infection experiments, PBMC were infected with $O$. tsutsugamushi, and then infected with R5 HIV-1 (which uses chemokine receptor CCR5 to infect the cell), X4 HIV-1 (which uses CXCR4), or R5X4 HIV-1 (which uses both CCR5 and CXCR4). Stimulation with $O$. tsutsugamushi consistently enhanced infection with X4 or R5X4 HIV-1, irrespective of subtypes B or E (Figure 

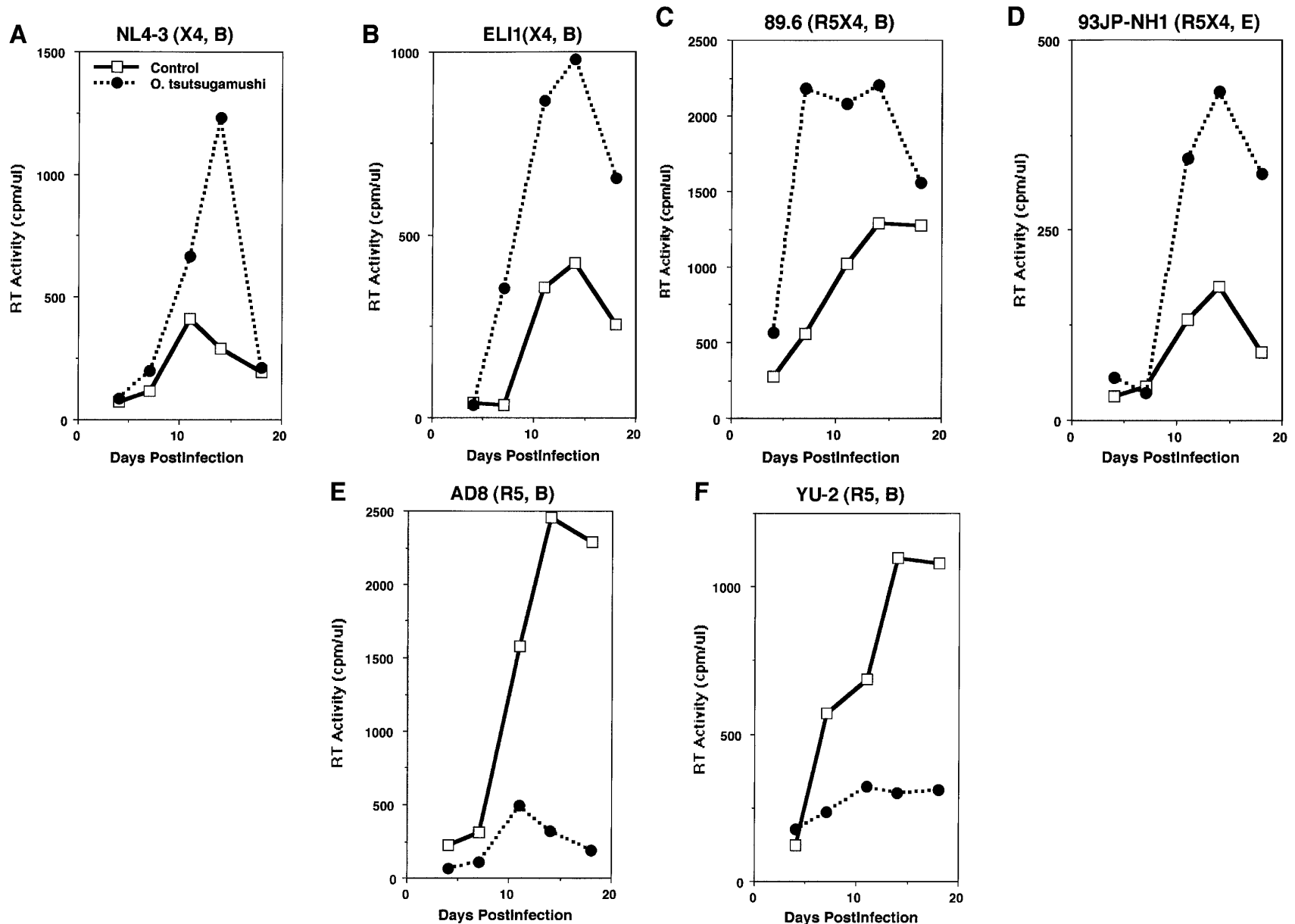

FIgURE 1. Dichotomous effects of Orientia tsutsugamushi on in vitro replication of human immunodeficiency virus-1 (HIV-1). Infection with O. tsutsugamushi enhanced replication of X4 HIV-1 (A, NL4-3 and B, ELI1) and R5X4 HIV-1 (C, 89.6 and D, 93JP-NH1), but suppressed replication of R5 HIV-1 (E, AD8 and F, YU-2). Peripheral blood mononuclear cells were uninfected (squares) or infected (circles) with the $O$. tsutsugamushi Karp strain for 24 hours prior to HIV-1 infection, and HIV-1 replication was monitored by reverse transcriptase (RT) assays. Similar results were obtained using the Kuroki strain of $O$. tsutsugamushi.

1A-D). However, the R5 HIV-1-inhibitory effect of $O$. tsutsugamushi was not consistent; it either suppressed (Figure 1E and $\mathrm{F}$ and donors 1,3 , and 5 in Table 1 ) or enhanced (donors 2 and 4 in Table 1) replication of R5 HIV-1. Cell viability of $O$. tsutsugamushi-stimulated cultures, as judged by trypan blue staining, was similar to that of unstimulated cultures. These results suggested that $O$. tsutsugamushi has several different activities against HIV-1 infection.

To more precisely delineate how O. tsutsugamushi can modulate HIV-1 infection, we performed single-round viral replication assays in which PBMC were uninfected or infected with $O$. tsutsugamushi prior to or after HIV-1 infection. In these assays, pretreatment with $O$. tsutsugamushi suppressed R5, but not X4 HIV-1 infection (Figure 2). In contrast, posttreatment enhanced HIV-1 infection, irrespective of coreceptor usage (Figure 2). Heat-inactivated O. tsutsugamushi had effects similar to those of live O. tsutsugamushi stocks (Figure 2). Thus, O. tsutsugamushi appeared to inhibit CCR5mediated cellular entry of R5 HIV-1 and facilitate the postentry viral replicative cycle. In addition, the productive replication of $O$. tsutsugamushi is not necessary for its effects.

Down-regulation of CCR5 expression by $O$. tsutsugamushi. Since the level of CCR5 expression correlates well with infectability of cells with R5 HIV-1, we hypothesized that $O$. tsutsugamushi infection leads to down-regulation of CCR5 expression. Flow cytometry studies have clearly proven our hypothesis: O. tsutsugamushi infection downregulated CCR5 expression (Figure 3 and Table 1) but had little effect on CXCR4 expression. ${ }^{6}$

Up-regulation of HIV-1 LTR activity by $O$. tsutsugamushi. Next, we investigated how $O$. tsutsugamushi infection en-

TABLE 1

CCR5 expression and infectability by R5 human immunodeficiency virus-1 (HIV-1) of peripheral blood mononuclear cells (PBMC) stimulated with Orientia tsutsugamushi*

\begin{tabular}{cccccc}
\hline & \multicolumn{2}{c}{ CCR5 expression (\%) } & & \multicolumn{2}{c}{ R5 HIV-1 infectability $(\mathrm{cpm} / \mu \mathrm{L})$} \\
\cline { 2 - 3 } \cline { 5 - 6 } Donor & Unstimulated & Stimulated & & Unstimulated & Stimulated \\
\hline 1 & 5.1 & $<0.5$ & & 2,460 & 480 \\
2 & 6.8 & 1.1 & & 1,980 & 4,230 \\
3 & 2.4 & $<0.5$ & & 2,690 & 140 \\
4 & 0.8 & $<0.5$ & & 120 & 710 \\
5 & 3.6 & $<0.5$ & & 2,360 & 220 \\
\hline
\end{tabular}

* Cell surface CCR5 expression was determined by flow cytometry 24 hours after stimulation of PBMC from five donors with the $O$. tsutsugamushi Karp strain. Peak reverse
transcriptase titers in infection of unstimulated or stimulated PBMC with HIV-1 AD8 are shown as R5 HIV-1 infectability. Results in donor 1 are also shown in Figures 1E and 3. 


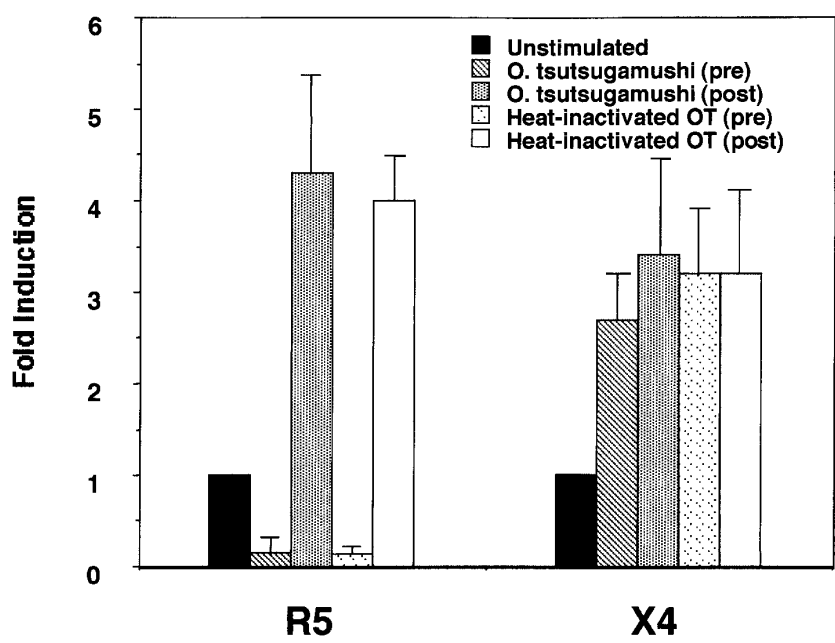

FIGURE 2. Differential effects of Orientia tsutsugamushi (OT) on R5 human immunodeficiency virus-1 (HIV-1) infection before and after cellular entry. Pretreatment with $O$. tsutsugamushi suppressed R5 HIV-1 infection, while post-treatment enhanced HIV-1 infection. A replication-incompetent luciferase-reporter recombinant virus pseudotyped with envelope (env) protein from R5 HIV-1 JRFL or X4 HIV-1 HXB2 was infected into peripheral blood mononuclear cells (PBMC). The PBMC were not treated, pretreated (pre), or post-treated (post) with the O. tsutsugamushi Karp strain (live or heat-inactivated), and luciferase activity in the infected cell lysates was determined. Fold induction indicates luciferase activity relative to that of a control (unstimulated) experiment. Results (mean \pm SD) of triplicate experiments are shown.

hanced post-entry HIV-1 replication. In transient expression assays using PBMC, HIV-1 LTR promoter activity from pGLHIV-1-LTR was markedly enhanced by stimulation with $O$. tsutsugamushi infection (Figure 4). Thus, increased viral replication resulted, at least in part, from up-regulation of viral transcription.

Mediation of the effects of $\boldsymbol{O}$. tsutsugamushi infection on HIV-1 infection through soluble activity. In good agreement with previous studies demonstrating that $O$. tsutsugamushi infection induces production of a number of cytokines including TNF- $\alpha$, interferon- $\gamma$ (IFN- $\gamma$ ), and $\beta$-chemokines, ${ }^{7,8}$ levels of TNF- $\alpha$, IFN- $\gamma$, and RANTES protein in cell-free supernatants of $O$. tsutsugamushi-infected PBMC were $645 \mathrm{pg} / \mathrm{mL}$, $170 \mathrm{pg} / \mathrm{mL}$, and 8,350 pg/mL, respectively, while uninfected cells released $<15.6 \mathrm{pg} / \mathrm{mL}$ of TNF- $\alpha,<15.6 \mathrm{pg} / \mathrm{mL}$ of IFN- $\gamma$, and $120 \mathrm{pg} / \mathrm{mL}$ of RANTES protein, respectively. Although proinflammatory cytokines such as TNF- $\alpha$ have been shown to transactivate HIV-1 LTR activity, IFN- $\gamma$ and RANTES protein have dichotomous effects on HIV-1 replication, depending upon cell types and/or virus strains. ${ }^{2}$ To demonstrate that soluble activity mediated by those cytokines plays a critical role in modulating HIV-1 infection of O. tsutsugamushiinfected PBMC, cell-free supernatants were collected from $O$. tsutsugamushi-infected PBMC. Autologous uninfected PBMC were treated with the supernatants along with minocycline $(5 \mu \mathrm{g} / \mathrm{mL})$ to prevent replication of $O$. tsutsugamushi prior to or after infection with HIV-1. Minocycline did not influence HIV-1 infection at the concentration used in this study. As shown in Figure 5, soluble factors derived from $O$. tsutsugamushi-infected cells had similar effects on HIV-1 infection to O. tsutsugamushi infection per se.

In vitro reactivation of $\mathrm{HIV}-1$ upon stimulation with $O$. tsutsugamushi. Finally, we tested whether stimulation with $O$. tsutsugamushi can induce HIV-1 replication from PBMC derived from HIV-1-infected individuals whose plasma viral RNA was undetectable after HAART. Apheresis to obtain PBMC was performed according to protocol approved by the National Institute of Allergy and Infectious Diseases Institutional Review Board (Bethesda, MD). The $\mathrm{CD}^{+} \mathrm{T}$ cells and HLA-DR ${ }^{+}$cells were depleted by Dynabeads (Dynal, Lake Success, NY) CD8 and HLA-DR, and there were less than $0.1 \%$ HLA-DR $^{+} \mathrm{CD}^{+}{ }^{+} \mathrm{T}$ cells (activated $\mathrm{CD} 4^{+} \mathrm{T}$ cells) in the remaining PBMC preparations. These cells did not release HIV-1 p24 antigen without any stimulation; however, stimulation with phytohemagglutinin plus IL-2 induced in vitro HIV-1 replication in all patients tested (Table 2). When these cells were stimulated with $O$. tsutsugamushi, HIV-1 replication was induced in two of the four patients tested (Table 2). Thus, O. tsutsugamushi stimulation appears to have capability of inducing HIV-1 replication in patients whose plasma viremia is undetectable.

\section{DISCUSSION}

Orientia tsutsugamushi is a gram-negative bacillus that is an obligate intracellular parasite, and is distributed in many Asian countries and Pacific islands. Since HIV-1 infection is
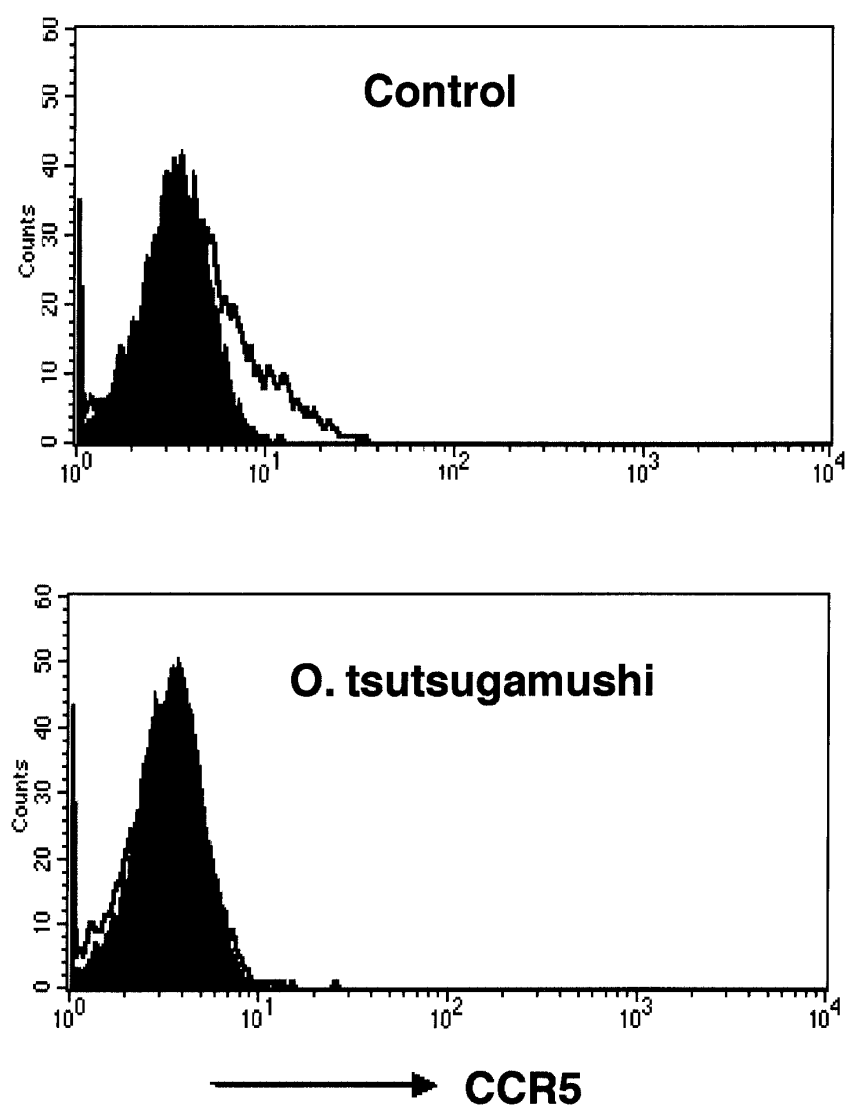

FIGURE 3. Down-regulation of expression of CCR5 by infection with Orientia tsutsugamushi. Peripheral blood mononuclear cells were infected with the Karp strain, and 24 hours later cell-surface expression of CCR5 was determined by flow cytometry using a monoclonal antibody (2D7) to CCR5. Staining with an isotype control (shaded areas) and with the monoclonal antibody to CCR5 (solid lines) are shown. 


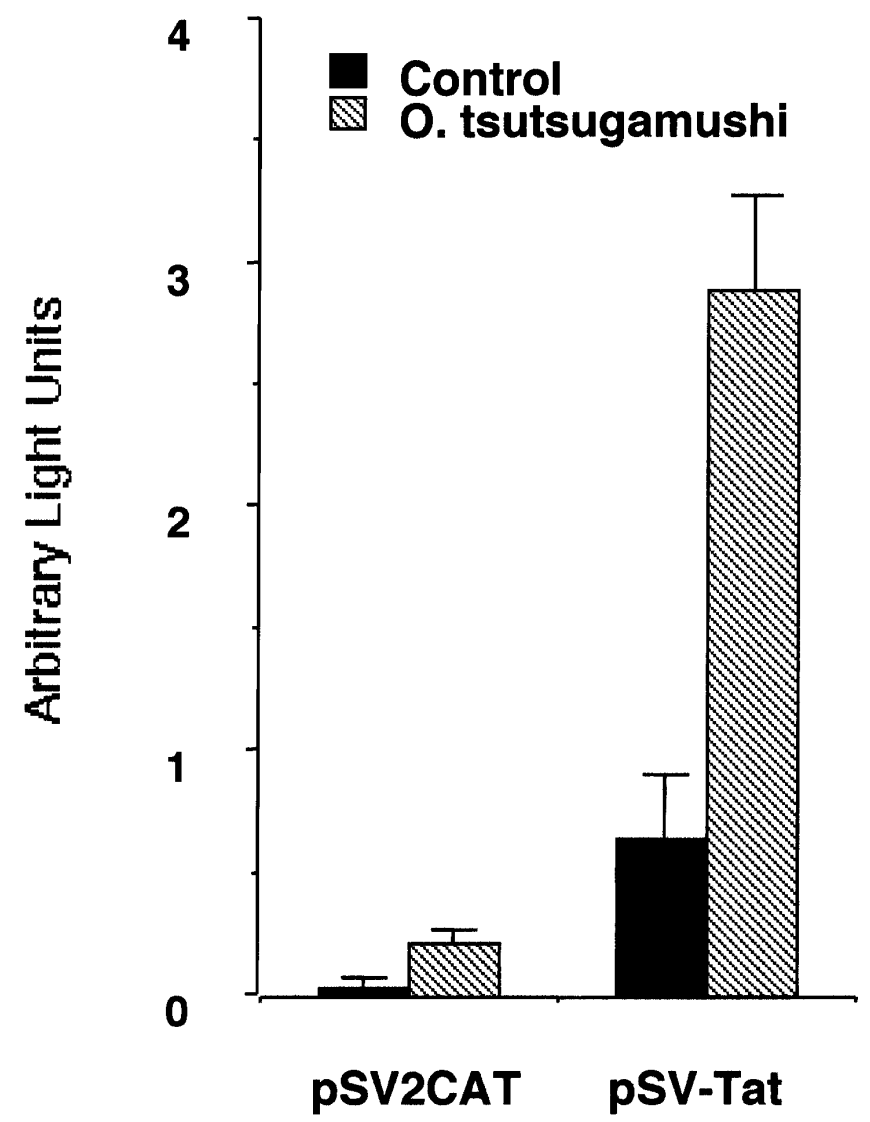

FIGURE 4. Up-regulation of human immunodeficiency virus-1 (HIV-1) long terminal repeat (LTR) activity by Orientia tsutsugamushi. Peripheral blood mononuclear cells were transfected with pGL-HIV-1-LTR along with pSV2CAT or pSV-Tat, and stimulated with $O$. tsutsugamushi. Luciferase activity in the transfected cell lysates was determined two days after transfection. Results (mean \pm $\mathrm{SD})$ of triplicate experiments are shown.

also endemic in some of these areas, interaction between the two microorganisms may have clinical significance. In this regard, a recent study from Thailand has reported that acute scrub typhus infection may suppress HIV-1 infection, ${ }^{1}$ which is in striking contrast to many other microbial coinfections that generally result in enhanced replication of HIV $-1 .{ }^{9}$ However, difficulty in conducting a prospective and wellcontrolled clinical study in Thailand, as well as the lack of precise laboratory data supporting their hypothesis, has resulted in substantial controversy.

Our in vitro study has suggested that $O$. tsutsugamushi infection had impacts on HIV-1 infection in several different ways, and that the net effect depends upon the balance of positive and negative factors. Difference in the net effects observed among different donors is intriguing and deserves further evaluation. Our preliminary data suggest that a number of host factors are involved in such variability (Moriuchi M, Moriuchi H, unpublished data). For example, while upregulation of HIV-1 expression from the LTR promoter secondary to proinflammatory cytokine production would benefit HIV-1 infection, down-regulation of CCR5 expression as well as production of chemokines would inhibit HIV-1 entry. Activation of LTR by microbial stimulation has been demonstrated in a variety of other co-infections. ${ }^{9}$ We have previously reported similar dichotomous effects of bacterial cell

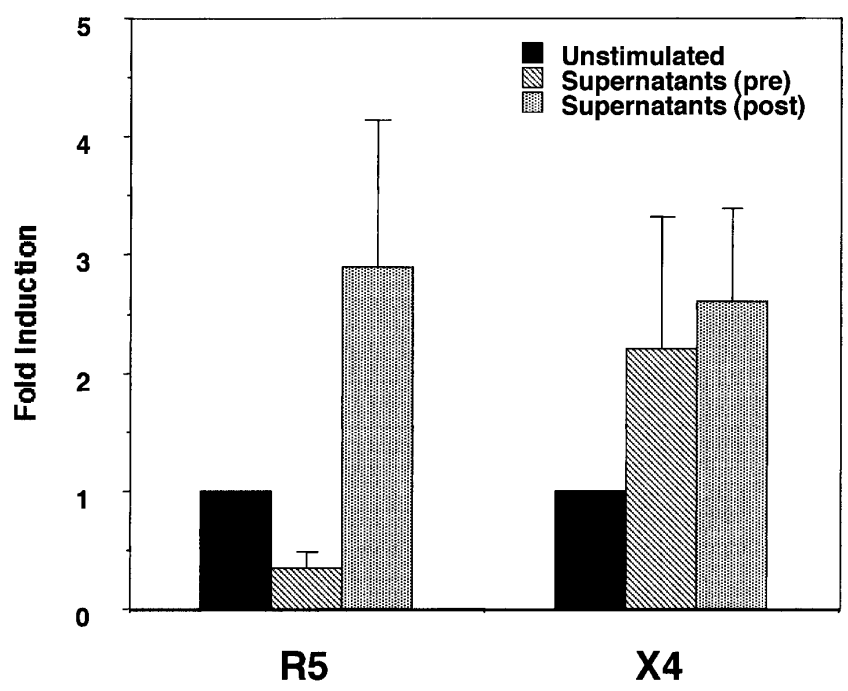

FIGURE 5. Mediation of the effects of infection with Orientia tsutsugamushi on human immunodeficiency virus-1 (HIV-1) infection through soluble activity. Peripheral blood mononuclear cells (PBMC) were infected with a replication-recombinant virus pseudotyped with envelope (env) protein from R5 HIV-1 JRFL or X4 HIV-1 HXB2. The PBMC were not treated, pretreated (pre), or post-treated (post) with supernatants from autologous PBMC infected with the $O$. tsutsugamushi Karp strain, and luciferase activity in the cell lysates was determined. Fold induction indicates luciferase activity relative to that of a control (untreated) experiment. Results (mean \pm SD) of triplicate experiments are shown.

wall components such as lipopolysaccharide (LPS) of gramnegative bacilli, lipoteichoic acid of gram-positive cocci, and lipoarabinomannan of mycobacteria on HIV-1 infection. ${ }^{2}$ Our results in the present study were not unexpected, since $O$. tsutsugamushi contains LPS in its cell wall structure, although rickettsial LPS generally has weaker endotoxin activity than that of other gram-negative bacilli. Thus, although it is still possible that in vivo infection with $O$. tsutsugamushi may have substantially distinct effects on HIV-1 infection, our in vitro study suggests that scrub typhus does not necessarily suppress HIV-1 infection and does have potential to enhance HIV-1 replication.

Interactions between HIV and other microbes do not appear to be simple. Recently, measles infection has been shown to suppress HIV viremia in patients, ${ }^{10}$ and we have also demonstrated that malaria parasites may suppress HIV-1 infec-

TABLE 2

In vitro reactivation of human immunodeficiency virus-1 (HIV-1) upon stimulation with Orientia tsutsugamushi*

\begin{tabular}{cccccc}
\hline & \multirow{2}{*}{$\begin{array}{c}\text { CD4+ T cells } \\
(/ \mu \mathrm{L})\end{array}$} & $\begin{array}{c}\text { HIV-1 RNA } \\
\text { copies }(/ \mathrm{mL})\end{array}$ & Unstimulated & O. tsutsugamushi & PHA/IL-2 \\
\hline 1 & 303 & $<50$ & $<7.8$ & $<7.8$ & 54 \\
2 & 238 & $<500$ & $<7.8$ & 27 & 55 \\
3 & 601 & $<500$ & $<7.8$ & $8.0 \dagger$ & 116 \\
4 & 596 & $<50$ & $<7.8$ & 39 & 64 \\
\hline
\end{tabular}

Peripheral blood mononuclear cells were derived from patients 1 through 43,4 whose plasma viremia was undetectable by the Amplicor Ultrasensitive HIV-1 Monitor assay
(Roche, Diagnostics Corporation, Indianapolis, IN) in patients 1 and 4 or bDNA assays (Roche, Diagnostics Corporation, Indianapolis, IN) in patients 1 and 4 or bDNA assays
(Chiron, Emeryville, CA) in patients 2 and 3 , were depleted of $\mathrm{CD}^{+}$cells and HLA-DR ${ }^{+}$ cells, and were either unstimulated or stimulated as indicated. Peak HIV-1 p24 titers that cells, and were either unstimulated or stimulated as indicated. Peak HIV-1 p24 titers that
were obtained on day 16 are shown. PHA = phytohemagglutinin, IL-2 = interleukin- 2 . $\dagger$ HIV-1 replication in the PBMC of patient 3 , as determined by slight increase in p 24 titer $\dagger$ HIV-1 replication in the PBMC of patient 3 , as determined
on day 16 , was not able to be expanded by further passages. 
tion in vitro. ${ }^{11}$ Further studies are required for better understanding of interaction between these microbes and HIV-1 in co-infected patients.

Received April 22, 2002. Accepted for publication January 20, 2003.

Acknowledgments: We thank A. S. Fauci for support, L. A. Ehler and S. B. Mizell for patient recruitment, and M. Yokoyama for technical assistance and graphic work.

Financial support: This work was supported in part by a grant provided by a Research for the Future Program (JSPS-RFTF97L00705) of the Japan Society for the Promotion of Science and by a Grantin-Aid from the Ministry of Education, Science, Sports and Culture, Japan.

Authors' addresses: Masako Moriuchi and Hiroyuki Moriuchi, Department of Molecular Microbiology and Immunology, Nagasaki University Graduate School of Biomedical Sciences, Nagasaki 8528523, Japan. Akira Tamura, Department of Microbiology, Niigata College of Pharmacy, Niigata 950-2076, Japan.

Reprint requests: Hiroyuki Moriuchi, Department of Pediatrics, Nagasaki University Hospital, 1-7-1 Sakamoto, Nagasaki 852-8501, Japan, Telephone: 81-95-849-7297, Fax: 81-95-849-7301, E-mail: hiromori@net.nagasaki-u.ac.jp

\section{REFERENCES}

1. Watt G, Kantipong P, de Souza M, Chanbancherd P, Jongsakul K, Ruangweerayud R, Loomis-Price LD, Polonis V, Myint KS, Birx DL, Brown AE, Krishna S, 2000. HIV-1 suppression during acute scrub-typhus infection. Lancet 356: 475-479.

2. Moriuchi M, Moriuchi H, Turner W, Fauci AS, 1998. Exposure to bacterial products renders macrophages highly susceptible to T-tropic human immunodeficiency virus type 1: implications for in vivo coinfections. J Clin Invest 102: 1540-1550.
3. Moriuchi H, Moriuchi M, Mizell SB, Ehler LA, Fauci AS, 2000. In vitro reactivation of HIV-1 from latently infected, resting CD4+ T cells upon bacterial stimulation. J Infect Dis 181: 2041-2044.

4. Moriuchi H, Moriuchi M, 2000. In vitro induction of HIV-1 replication in resting $\mathrm{CD}^{+} \mathrm{T}$ cells derived from individuals with undetectable plasma viremia upon stimulation with human Tcell leukemia virus type I. Virology 278: 514-519.

5. Tamura A, Urakami H, Tsuruhara T, 1988. Purification of Rickettsia tsutsugamushi by Percoll density gradient centrifugation. Microbiol Immunol 26: 321-328.

6. Shalekoff S, Tiemessen CT, 2001. Duration of sample storage dramatically alters expression of the human immunodeficiency virus coreceptors CXCR4 and CCR5. Clin Diagn Lab Immunol 8: 432-436.

7. Iwasaki H, Takada N, Nakamura T, Ueda T, 1997. Increased levels of macrophage colony-stimulating factor, gamma interferon, and tumor necrosis factor alpha in sera of patients with Orientia tsutsugamushi infection. J Clin Microbiol 35: 33203322.

8. Cho NH, Seong SY, Huh MS, Han TH, Koh YS, Choi MS, Kim IS, 2000. Expression of chemokine genes in murine macrophages infected with Orientia tsutsugamushi. Infect Immun 68: 594-600.

9. Blanchard A, Montagnier L, Gougeon ML, 1997. Influence of microbial infections on the progression of HIV disease. Trends Microbiol 5: 326-331.

10. Moss WJ, Ryon JJ, Monze M, Cutts F, Quinn TC, Griffin DE, 2002. Suppression of human immunodeficiency virus replication during acute measles. J Infect Dis 185: 1035-1042.

11. Moriuchi M, Moriuchi H, Mon HM, Kanbara H, Dichotomous effects of Plasmodium falciparum antigens on expression of human immunodeficiency virus (HIV) co-receptors on and infectability by HIV of CD4+ T cells. J Infect Dis 186: 1194-1197. 